\title{
Tunable surface plasmon-polariton resonance in organic light-emitting devices based on corrugated alloy electrodes
}

\author{
Xue-Mei Wen ${ }^{1}$, Yan-Gang Bi ${ }^{1 *}$, Fang-Shun $\mathrm{Yi}^{1}$, Xu-Lin Zhang ${ }^{1}$, \\ Yue-Feng Liu ${ }^{1}$, Wen-Quan Wang ${ }^{3}$, Jing Feng ${ }^{1 *}$ and Hong-Bo Sun ${ }^{1,2 *}$
}

\begin{abstract}
We report a feasible method to realize tunable surface plasmon-polariton (SPP) resonance in organic light-emitting devices (OLEDs) by employing corrugated Ag-Al alloy electrodes. The excited SPP resonance induced by the periodic corrugations can be precisely tuned based on the composition ratios of the Ag-Al alloy electrodes. With an appropriate composition ratio of the corrugated alloy electrode, the photons trapped in SPP modes are recovered and extracted effectively. The $25 \%$ increasement in luminance and $21 \%$ enhancement in current efficiency have been achieved by using the corrugated Ag-Al alloy electrodes in OLEDs.
\end{abstract}

Keywords: organic light-emitting devices; alloy electrodes; tunable surface plasmon-polariton resonance; periodic corrugation; light extraction

Wen XM, Bi YG, Yi FS, Zhang XL, Liu YF et al. Tunable surface plasmon-polariton resonance in organic light-emitting devices based on corrugated alloy electrodes. Opto-Electron Adv 4, 200024 (2021).

\section{Introduction}

Organic light-emitting devices (OLEDs), with the advantages of wide material selection, broadband spectra, high brightness, thin thickness, wide viewing angle, transparency and flexibility ${ }^{1-5}$, have become a research hotspot and have demonstrated their potential applications in flexible and stretchable equipment ${ }^{6,7}$. Nearly $100 \%$ of the internal quantum efficiency has been achieved in phosphorescent OLEDs, however, a great number of photons generated in active layers are trapped and lost in OLEDs. The power loss in OLEDs is derived from the substrate mode, waveguide mode, surface plas- mon-polariton (SPP) mode and so on ${ }^{8,9}$. The substrate mode induced by the reflection of the glass substrate/air interface can be suppressed by modifying the back side of the substrate ${ }^{10,11}$. For example, Tang et al. successfully released the trapped energy in substrate mode by applying quasi-periodic nanostructure arrays ${ }^{12}$. The commonly used ITO transparent electrode, with high refractive index, can cause the waveguide mode to trap photons inside OLEDs. Using novel transparent electrodes, such as metal nanowires ${ }^{13,14}$, ultrathin metal films ${ }^{15,16}$, and metal meshes ${ }^{17,18}$ to replace ITO can effectively recover photons trapped in waveguide mode. SPP mode,

${ }^{1}$ State Key Lab of Integrated Optoelectronics, College of Electronic Science and Engineering, Jilin University, Changchun 130012, China; ${ }^{2 S t a t e}$ Key Lab of Precision Measurement Technology and Instruments, Department of Precision Instrument, Tsinghua University, Beijing 100084, China; ${ }^{3}$ College of Physics, Jilin University, Changchun 130012, China.

"Correspondence: YG Bi, E-mail: yangang_bi@jlu.edu.cn; J Feng, E-mail: jingfeng@jlu.edu.cn; HB Sun, E-mail: hbsun@tsinghua.edu.cn

Received: 29 June 2020; Accepted: 20 September 2020; Published: 25 August 2021

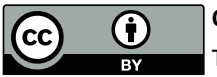

Open Access This article is licensed under a Creative Commons Attribution 4.0 International License.

To view a copy of this license, visit http://creativecommons.org/licenses/by/4.0/.

(C) The Author(s) 2021. Published by Institute of Optics and Electronics, Chinese Academy of Sciences. 
associated with the planar metal/dielectric interface in OLEDs, is non-radiative because of the mismatch of momenta between SPP and free space photon ${ }^{19}$. Periodic corrugations are used to excite SPP resonance, and corrugated metal electrodes can extract the photons trapped by SPP mode in OLEDs ${ }^{20}$. The period of corrugations is a key factor to excite SPP resonance at the desired lightemitting wavelength in various OLEDs $^{21,22}$. Two-beam interference lithography ${ }^{21,23}$, nanoimprint lithography $(\mathrm{NIL})^{20,24}$, electron beam lithography ${ }^{25}$, and focused ion beam lithography ${ }^{26}$ are common technologies to fabricate periodic corrugations. However, the complex fabrication process with high cost make them difficult in commercial applications to obtain corrugations with different periods in various OLEDs.

The wavelength of excited SPP resonance can also be modified by material properties of the metal/dielectric interface ${ }^{27,28}$. In this letter, we propose to excite a tunable $\mathrm{SPP}$ resonance in OLEDs based on corrugated Ag- $\mathrm{Al}$ alloy electrode with the fixed periodicity. We use NIL to introduce periodic corrugations on substrates and a codeposition method to obtain Ag-Al alloy electrodes in OLEDs. By changing the deposition rate of $\mathrm{Ag}$ and $\mathrm{Al}$, we modify the composition ratio of the alloy electrodes. The SPP mode can be effectively excited by the corrugated alloy electrodes and accurately tuned according to the composition ratio. We fix the period of corrugation at $290 \mathrm{~nm}$, and choose the deposition rates of $\mathrm{Ag}$ and $\mathrm{Al}$ to be $0.16 \mathrm{~nm} / \mathrm{s}$ and $0.016 \mathrm{~nm} / \mathrm{s}$, respectively, and the excited SPP resonance by the corrugated alloy electrode is adjusted to the desired light-emitting wavelength of 564 $\mathrm{nm}$. Compared with the planar devices, the luminance and efficiency of corrugated OLEDs are effectively improved owing to the recovery and extraction of trapped photons in SPP.

\section{Material and method}

\section{Fabrication of periodic corrugations}

The fabrication process of the periodic corrugations based on NIL is described in Fig. 1. The Si template with $290 \mathrm{~nm}$ periodic corrugations was cleaned with acetone and ethanol for three times at $75{ }^{\circ} \mathrm{C}$, and then was covered by polydimethylsiloxane (PDMS) as shown in Fig. 1(a) and 1(b). In order to remove bubbles between the $\mathrm{Si}$ template and the PDMS, the sample was placed in a vacuum chamber for $30 \mathrm{~min}$. After the PDMS was thermally solidified and peeled off, the corrugated structures on the Si template were transferred to the PDMS film (Fig. 1(c) and 1(d)).

SU-8 2025 photoresist (MicroChem Corp.) was diluted with cyclopentanone to $0.1 \mathrm{~g} / \mathrm{mL}$, and then spincoated on a precleaned glass substrate at $2000 \mathrm{rpm}$ for $30 \mathrm{~s}$ (Fig. 1(e)). The substrate was dried in an oven at $95{ }^{\circ} \mathrm{C}$ for $30 \mathrm{~min}$. The SU- 8 coated glass substrate was covered by the prepared PDMS template with corrugations (Fig. $1(\mathrm{f})$ ), and then placed in a chamber of the NIL system (CNI1-5vac, NIL Technology Company). After the NIL process for $20 \mathrm{~min}$ at 5 bar and $100{ }^{\circ} \mathrm{C}$, the corrugations with period of $290 \mathrm{~nm}$ were transferred to the SU-8 film (Fig. 1(g) and 1(h)). Finally, the corrugated SU-8 was exposed under ultraviolet (UV) for $1 \mathrm{~min}$ to solidify.

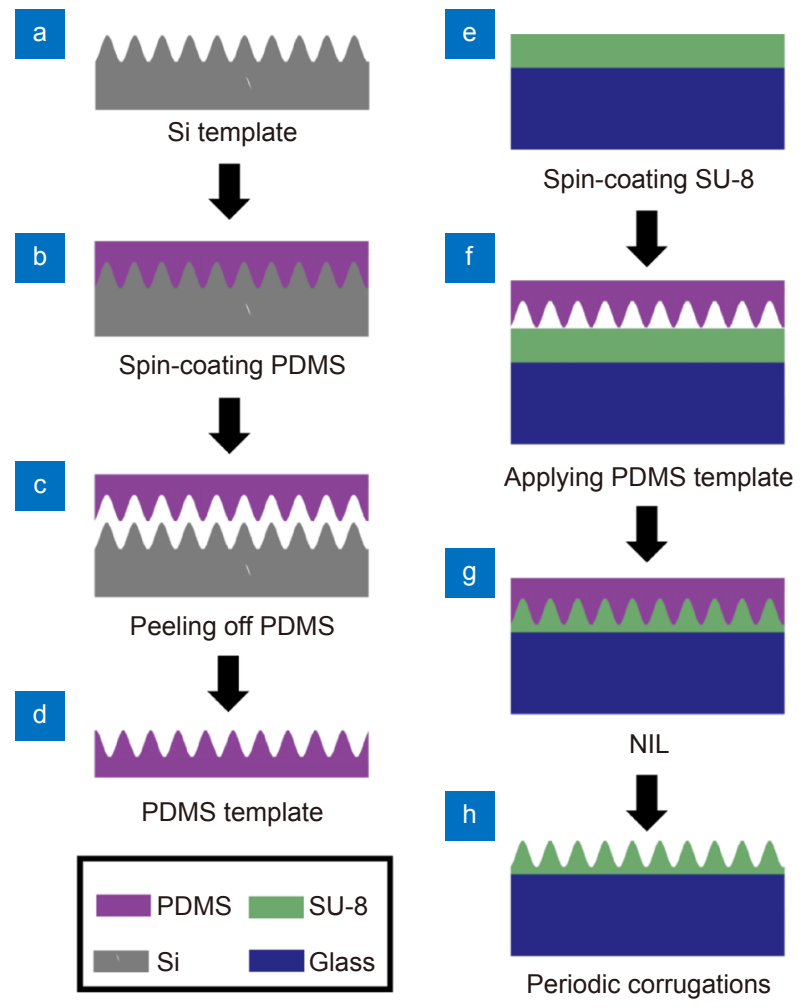

Fig. 1 | Fabrication process of the periodic corrugations based on NIL. (a) Si template with $290 \mathrm{~nm}$ periodic corrugations. (b) PDMS was spin-coated on Si template. (c) Solidified PDMS film was peeled off. (d) The corrugations were transferred to PDMS template. (e) SU8 was spin-coated on a pre-cleaned glass substrate. (f) Prepared PDMS template was placed on the SU-8 film. (g) NIL processes were applied. (h) The periodic corrugations were prepared on the substrate.

\section{Fabrication of Ag-Al alloy electrodes and OLEDs}

$\mathrm{Ag}-\mathrm{Al}$ alloy electrodes and OLEDs were obtained by physical vapor deposition. The glass substrates covered by SU-8 film were placed in a vacuum chamber. $3 \mathrm{~nm}$ $\mathrm{MoO}_{3}$ and $8 \mathrm{~nm} \mathrm{Au}$ were first deposited on the substrate as a transparent ultrathin metal anode, and $\mathrm{MoO}_{3}$ served 
as a seed layer to suppress the Volmer-Weber growth of $\mathrm{Au}^{29}$. Subsequently, $3 \mathrm{~nm} \mathrm{MoO}_{3}, 40 \mathrm{~nm} \mathrm{~N}, \mathrm{~N}^{\prime}$-DiphenylN,N'-bis(1,1'-biphenyl)-4,4'-diamine (NPB), $30 \mathrm{~nm} \mathrm{4,4'-}$ Bis(Ncarbazolyl)-1,1'-biphenyl (CBP) doped with $5 \mathrm{wt} \%$ 2,3,5,6-tetrakis(3,6-diphenylcarbazol-9-yl)-1,4-dicyanobenzene $\left(\operatorname{Ir}(\mathrm{BT})_{2}(\mathrm{acac})\right), 30 \mathrm{~nm}$ 2,2',2"(1,3,5-Nbenzenetriyl)tris-[1-phenyl-1Hbenzimidazole] (TpBi), and 3 $\mathrm{nm}$ Ca were deposited ${ }^{18}$. Finally, Ag and Al were co-deposited to obtain the alloy cathode with the thickness of $80 \mathrm{~nm}$. The deposition rate of Ag was fixed at $0.16 \mathrm{~nm} / \mathrm{s}$, the deposition rate of $\mathrm{Al}$ was changed to modify the composition ratio of the alloy electrode.

The absorption spectra of corrugated OLEDs was measured by a UV-Vis spectrophotometer (UV-2550, SHIMADZU), and a rotatable sample holder was fixed in the UV-Vis spectrophotometer to measure the angle-dependent absorption spectra. The electroluminescence (EL) performance of OLEDs were characterized by a Keithley 2400 Source Meter and PR-655 spectroradiometer (Photo Research Inc.). In order to detect the angle-dependent EL properties of OLEDs, the devices were fixed on a rotating stage and PR-655 spectroradiometer was set in the vertical direction.

\section{Numerical simulation}

The Finite-difference time-domain (FDTD) method was applied for numerical simulation. The metal and alloy electrodes were based on Drude model. The refractive indices of the organic materials in OLEDs were experimentally measured by ellipsometry. A modulated Gaus-
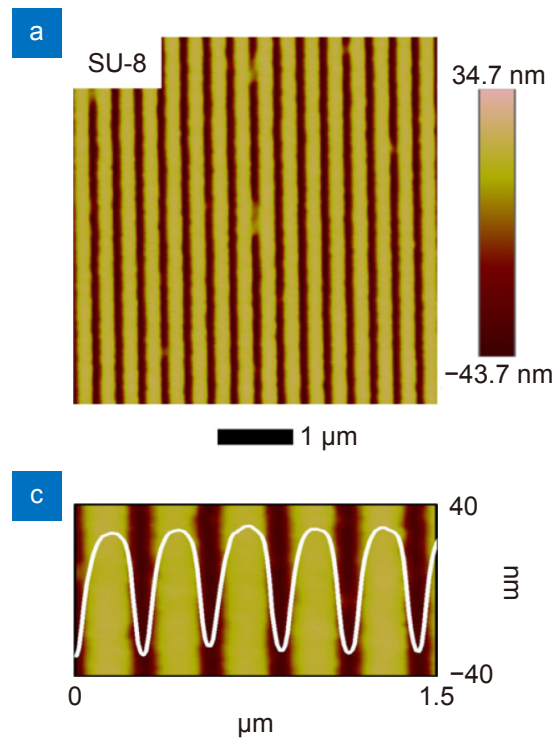

sian pulse was applied as the incident light. Periodic boundary conditions and perfectly matched layers were set for the FDTD simulation.

\section{Results and discussion}

NIL process was used to transfer the corrugations with a period of $290 \mathrm{~nm}$ from a Si template to the SU-8 film on the substrate, with PDMS as the transfer medium. The surface morphology of the corrugated SU-8 film was characterized by AFM, and the periodic corrugation prepared by NIL has a uniform morphology with a precise period of $290 \mathrm{~nm}$ and a neat height of $55 \mathrm{~nm}$ (Fig. 2(a) and $2(\mathrm{~b}))$. After the corrugation was prepared on the SU8-coated substate, an ultrathin Au electrode and an OLED were thermally deposited. The surface morphologies of the periodic corrugations before and after the deposition of ultrathin Au film are compared in Fig. 2. The corrugations exhibit the fixed period and amplitude without obvious changes of the surface morphology. The AFM images and the height profiles demonstrate that the thermal deposition process cannot damage the corrugated SU-8 films, and the corrugations can be expected to copy and transfer to each functional layers of OLEDs during the subsequent deposition process.

To effectively improve the photons extraction of the OLEDs, the excited SPP resonance should match the light-emitting wavelength of OLEDs. The emission peak of $\operatorname{Ir}(\mathrm{BT})_{2}$ (acac)-based OLEDs is located at $564 \mathrm{~nm}^{20}$. The wavelength of excited SPP resonance at the metal

\section{b}
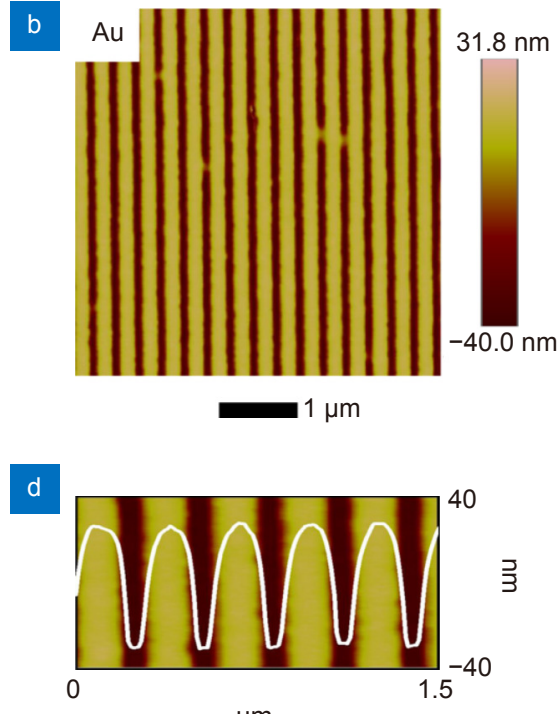

$\mu \mathrm{m}$

Fig. 2 | The AFM images of corrugations (a) prepared on glass substrate by NIL and (b) after deposition of ultrathin Au electrode. (c) and (d) are the height profiles of corrugations on the substrate and Au electrode, respectively. The periodic corrugations before and after deposition of Au film show consistent surface morphologies. 
cathode/organic materials interface was determined by measuring the enhanced absorption spectra induced by the corrugations in the corrugated OLEDs. Planar devices without corrugations were used as the reference to eliminate the absorption of materials, and the peaks in the absorption spectra come from the SPP resonance excited by the corrugations ${ }^{30}$. Figure $3(\mathrm{a})$ shows the enhanced absorption spectra of OLEDs with various metal cathodes. The wavelength of the excited SPP mode associated with $\mathrm{Al}, \mathrm{Ag}, \mathrm{Au}$, and $\mathrm{Cu}$ metal cathodes are 518 $\mathrm{nm}, 583 \mathrm{~nm}, 632 \mathrm{~nm}$, and $621 \mathrm{~nm}$, respectively. FDTD method has been applied to simulate the SPP resonance, which demonstrates a consistent result in Fig. 3(b). Considering the desired outcoupling wavelength of $564 \mathrm{~nm}$, as well as the cost and stability of metals, we choose Ag and $\mathrm{Al}$ to obtain the alloy electrode. A broader tunable wavelength range of the excited SPP resonance can be expected based on Al-Au alloy electrode.

Ag-Al alloy cathodes with different composition ra-

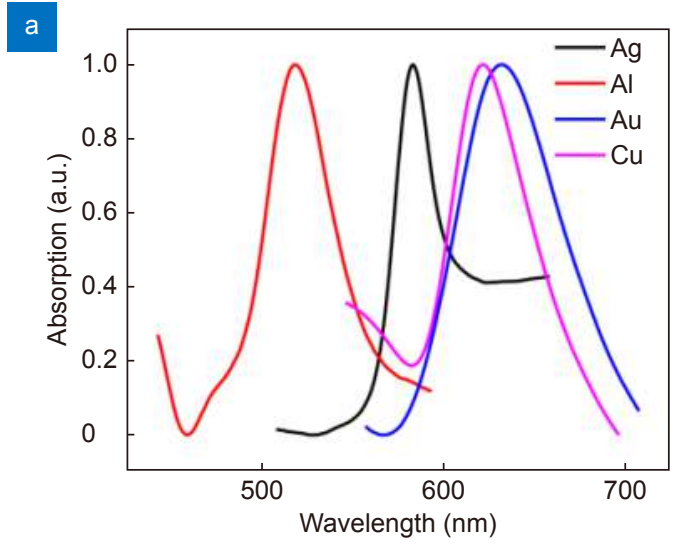

tios can be easily obtained by changing the deposition rate of $\mathrm{Ag}$ and $\mathrm{Al}$ during the co-deposition process. We controlled the deposition rate of $\mathrm{Ag}$ at $0.16 \mathrm{~nm} / \mathrm{s}$, and the deposition rate of $\mathrm{Al}$ was adjusted to $0.008 \mathrm{~nm} / \mathrm{s}, 0.016$ $\mathrm{nm} / \mathrm{s}$, and $0.024 \mathrm{~nm} / \mathrm{s}$. As a result, the Ag: Al composition molar ratios of the alloy electrodes were about $0.952: 0.048,0.909: 0.091$, and $0.870: 0.130$, respectively. The enhanced absorption spectra of corrugated OLEDs based on alloy cathodes with various composition ratios are shown in Fig. 4(a). As the Ag composition ratio decreases in the alloy, the enhanced absorption peak gradually blue-shifts from $583 \mathrm{~nm}$, corresponding to the Ag-cathode-based OLEDs, to $518 \mathrm{~nm}$ associated with Al-cathode-based devices. The enhanced absorption peaks of OLEDs based on $\mathrm{Ag}_{0.952} \mathrm{Al}_{0.048}$, $\mathrm{Ag}_{0.909} \mathrm{Al}_{0.091}$, and $\mathrm{Ag}_{0.870} \mathrm{Al}_{0.130}$ alloy cathodes are 572 $\mathrm{nm}, 564 \mathrm{~nm}$, and $555 \mathrm{~nm}$, respectively, and the corrugated $\mathrm{Ag}_{0.909} \mathrm{Al}_{0.091}$ alloy electrode induced SPP resonant peak is in accordance with the light-emitting wavelength

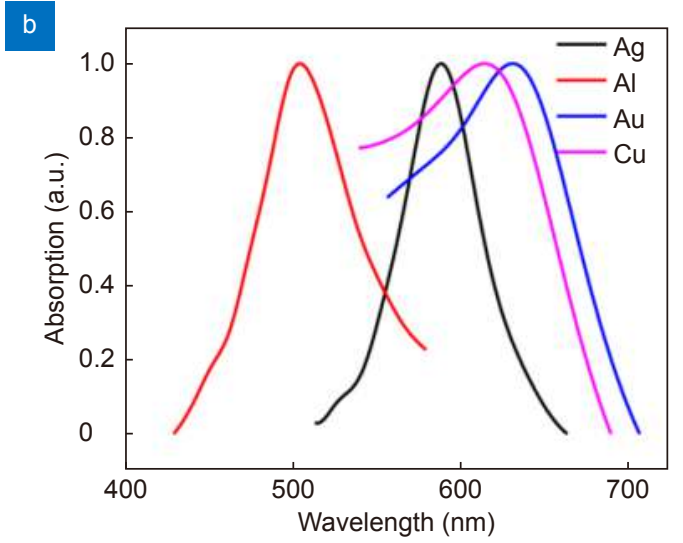

Fig. 3 | (a) Measured and (b) simulated absorption spectra of OLEDs based on Ag, Al, Au, Cu metal cathodes
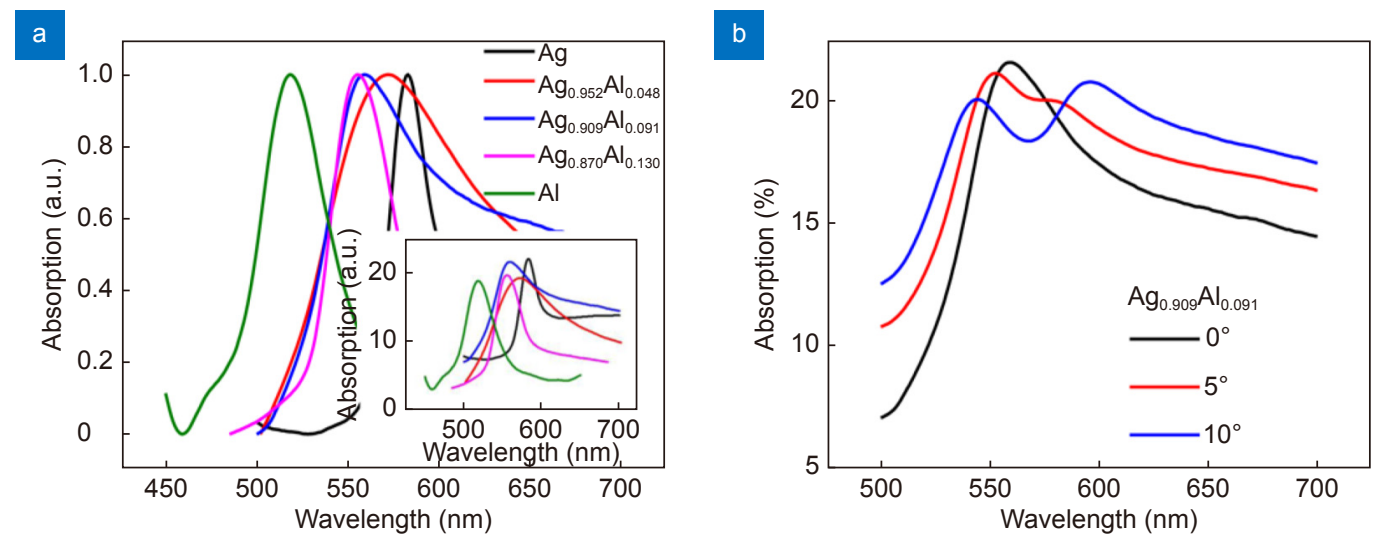

Fig. 4 | (a) The normalized absorption spectra of OLEDs based on $\mathrm{Ag}$ cathode (black line), $\mathrm{Al}$ cathode (green line), $\mathrm{Ag}_{0.952} \mathrm{Al}_{0.048}$ alloy cathode (red line), $\mathrm{Ag}_{0.909} \mathrm{Al}_{0.091}$ alloy cathode (blue line), and $\mathrm{Ag}_{0.870} \mathrm{Al}_{0.130}$ alloy cathode (magenta line), respectively. (b) The angle-dependent absorp-

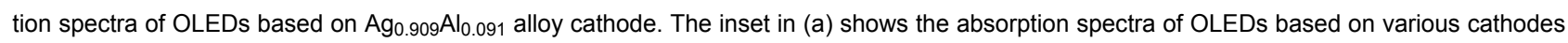
without normalization. 
of $\operatorname{Ir}(\mathrm{BT})_{2}(\mathrm{acac})$. To further confirm the enhanced absorption peaks arising from the excited SPP mode, the angle-dependent absorption spectra of $\mathrm{Ag}_{0.909} \mathrm{Al}_{0.091^{-}}$ based OLEDs were measured in Fig. 4(b). The absorption peak splits when the angle changes and the split peaks shift with angles, which is consistent with the characteristics of SPP resonant peak. It can be concluded that the corrugated Ag-Al alloy electrode, with composition molar ratio of $0.909(\mathrm{Ag}): 0.091(\mathrm{Al})$ and period of 290 $\mathrm{nm}$, can effectively excite the SPP mode in OLEDs at the desired light-emitting wavelength of $564 \mathrm{~nm}$.

The EL performances of OLEDs with corrugated $\mathrm{Ag}_{0.909} \mathrm{Al}_{0.091}$ alloy cathode are summarized in Fig. 5. Planar OLEDs with the same alloy electrodes are also compared. By introducing the plasmonic corrugations with period of $290 \mathrm{~nm}$, the luminance and efficiency of OLEDs have been obviously improved. Luminance is enhanced from $74810 \mathrm{~cd} / \mathrm{m}^{2}$ for planar OLEDs to 93320 $\mathrm{cd} / \mathrm{m}^{2}$ for the corrugated devices, and current efficiency is improved from $32.63 \mathrm{~cd} / \mathrm{A}$ to $39.46 \mathrm{~cd} / \mathrm{A}$, resulting in the enhancement of $25 \%$ in luminance and $21 \%$ in current efficiency, respectively. The corrugated OLEDs ex- hibits a higher current density than planar devices, and the corrugated device operated at $6 \mathrm{~V}$ demonstrates the higher brightness than the planar device from the photos in the inset of Fig. 5(a). OLEDs with and without corrugations have almost the same EL spectra as shown in Fig. 5(c). The angle-dependent EL spectra of the corrugated OLEDs were further measured under TM polarization. It can be clearly observed from Fig. 5(d) that the EL spectra changes significantly at various observation angles. The emission peak of $564 \mathrm{~nm}$ at $0^{\circ}$ gradually widens, splits and shifts with the increase of angles, which is consistent with the angle-dependent absorption spectra in Fig. 4(b). From the above experimental results, we can confirm that the EL performance of OLEDs can be effectively increased by introducing periodic corrugations, and the improvements arise from the out-coupling of SPP mode which is excited at the desired lightemitting wavelength by the corrugated $\mathrm{Ag}-\mathrm{Al}$ alloy electrode with an appropriate composition molar ratio.

\section{Conclusions}

In summary, we design a corrugated Al-Ag alloy a
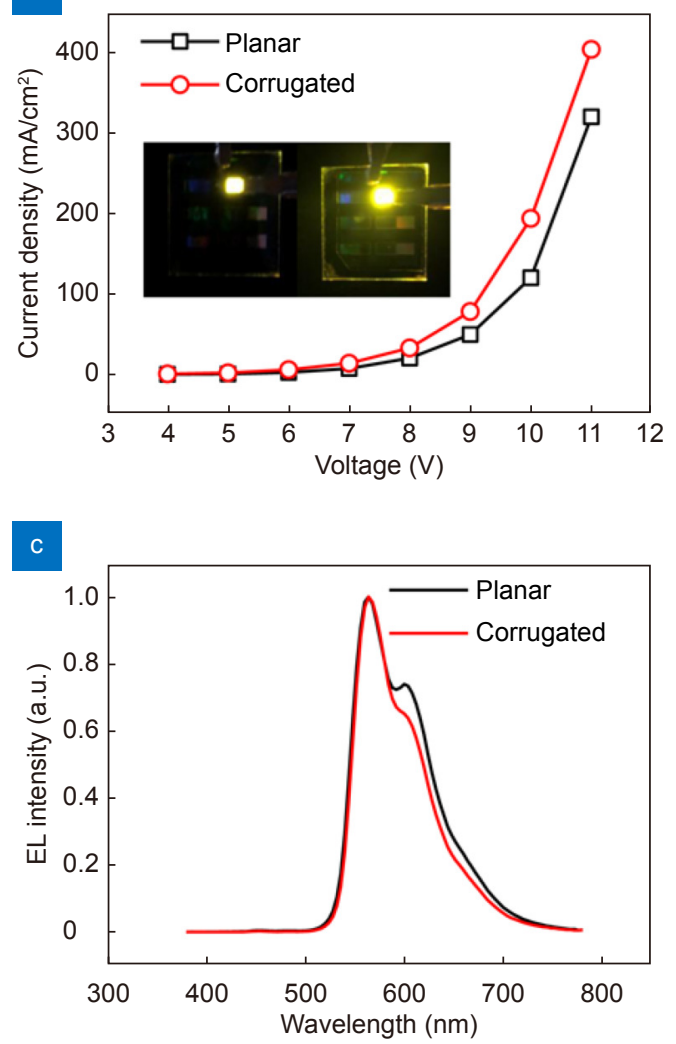

b

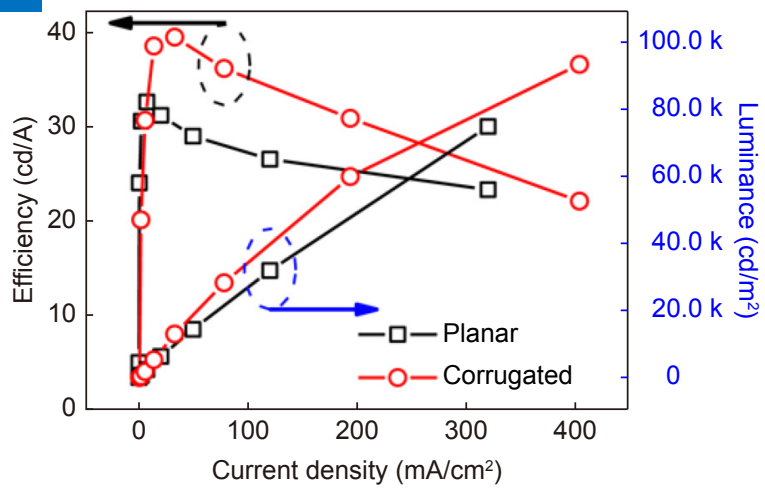

d

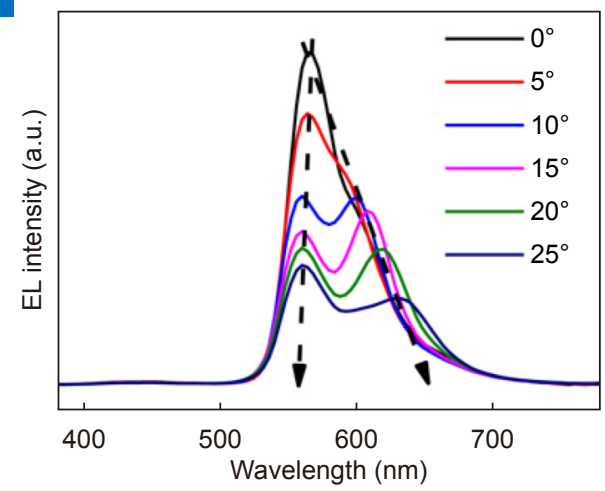

Fig. 5 | EL performance of OLEDs based on Ag-Al alloy cathodes with and without corrugations. (a) Current density-voltage and (b) efficiency-current density-luminance features, and (c) normalized EL spectra of corrugated and planar OLEDs. (d) The angle-dependent EL spectra of the corrugated OLEDs under TM polarization. The inset in (a) shows the photos of corrugated OLEDs and planar OLEDs operated at 6 V. 
electrode with period of $290 \mathrm{~nm}$ in OLEDs based on nanoimprint lithography and co-deposition technologies to excite a tunable SPP mode. By modifying the deposition rates of $\mathrm{Ag}$ and $\mathrm{Al}$ to change the composition molar ratios of the alloy cathodes, a tunable SPP resonance in corrugated OLEDs has been realized without changing the period of the corrugations. The resonant wavelength of the excited SPP mode, induced by the corrugated $\mathrm{Ag}_{0.909} \mathrm{Al}_{0.091}$ alloy cathode in OLEDs, is exactly consistent with the emission peak of the OLEDs, and the photons trapped in SPP mode are effectively out-coupled and extracted. Due to the improved light extraction in OLEDs, the luminance and current efficiency of the OLEDs with corrugated $\mathrm{Ag}_{0.909} \mathrm{Al}_{0.091}$ alloy cathode have been increased by $25 \%$ and $21 \%$, respectively.

\section{References}

1. Liu LH, Li SL, Wu L, Chen DF, Cao K et al. Enhanced flexibility and stability of PEDOT:PSS electrodes through interfacial crosslinking for flexible organic light-emitting diodes. Org Electron 89, 106047 (2021).

2. Jeon Y, Choi HR, Kwon JH, Choi S, Nam KM et al. Sandwichstructure transferable free-form OLEDs for wearable and disposable skin wound photomedicine. Light Sci Appl 8, 114 (2019).

3. Yin D, Chen ZY, Jiang NR, Liu YF, Bi YG et al. Highly transparent and flexible fabric-based organic light emitting devices for unnoticeable wearable displays. Org Electron 76, 105494 (2020).

4. Ding R, Dong FX, An MH, Wang XP, Wang MR et al. High-color-rendering and high-efficiency white organic light-emitting devices based on double-doped organic single crystals. Adv Funct Mater 29, 1807606 (2019).

5. Huang YG, Hsiang EL, Deng MY, Wu ST. Mini-LED, MicroLED and OLED displays: present status and future perspectives. Light Sci App/ 9, 105 (2020).

6. Song MG, Kim KS, Yang HI, Kim SK, Kim JH et al. Highly reliable and transparent $\mathrm{Al}$ doped $\mathrm{Ag}$ cathode fabricated using thermal evaporation for transparent OLED applications. Org Electron 76, 105418 (2020).

7. Yin D, Jiang NR, Liu YF, Zhang XL, Li AW et al. Mechanically robust stretchable organic optoelectronic devices built using a simple and universal stencil-pattern transferring technology. Light Sci Appl 7, 35 (2018).

8. Baek K, Lee DM, Lee YJ, Choi H, Seo J et al. Simultaneous emission of orthogonal handedness in circular polarization from a single luminophore. Light Sci App/ 8, 120 (2019).

9. Feng J, Liu YF, Bi YG, Sun HB. Light manipulation in organic light-emitting devices by integrating micro/nano patterns. Laser Photonics Rev 11, 1600145 (2017).

10. Qu Y, Kim J, Coburn C, Forrest SR. Efficient, nonintrusive outcoupling in organic light emitting devices using embedded microlens arrays. ACS Photonics 5, 2453-2458 (2018).

11. Salehi A, Fu XY, Shin DH, So F. Recent advances in OLED optical design. Adv Funct Mater 29, 1808803 (2019).

12. Zhou L, Ou QD, Li YQ, Xiang HY, Xu LH et al. Efficiently releasing the trapped energy flow in white organic light-emitting diodes with multifunctional nanofunnel arrays. Adv Funct Mater 25, 2660-2668 (2015).

13. Choi J, Shim YS, Park $\mathrm{CH}$, Hwang $\mathrm{H}$, Kwack JH et al. Junction-free electrospun ag fiber electrodes for flexible organic light-emitting diodes. Small 14, 1702567 (2018).

14. Putnin T, Lertvachirapaiboon C, Ishikawa R, Shinbo K, Kato K et al. Enhanced organic solar cell performance: Multiple surface plasmon resonance and incorporation of silver nanodisks into a grating-structure electrode. Opto-Electron Adv 2, 190010 (2019).

15. Bi YG, Liu YF, Zhang XL, Yin D, Wang WQ et al. Ultrathin metal films as the transparent electrode in ITO-free organic optoelectronic devices. Adv Opt Mater 7, 1800778 (2019).

16. Morales-Masis M, De Wolf S, Woods-Robinson R, Ager JW, Ballif C. Transparent electrodes for efficient optoelectronics. Adv Electron Mater 3, 1600529 (2017).

17. Ho MD, Liu YY, Dong DS, Zhao YM, Cheng WL. Fractal gold nanoframework for highly stretchable transparent strain-insensitive conductors. Nano Lett 18, 3593-3599 (2018).

18. Yi FS, Bi YG, Zhang XL, Yin D, Liu YF et al. Highly flexible and mechanically robust ultrathin Au grid as electrodes for flexible organic light-emitting devices. IEEE Trans Nanotechnol 18, 776-780 (2019).

19. Barnes WL, Dereux A, Ebbesen TW. Surface plasmon subwavelength optics. Nature 424, 824-830 (2003).

20. Ma C, Gao XM, Bi YG, Yi FS, Zhang XL et al. Directly imprinted periodic corrugation on ultrathin metallic electrode for enhanced light extraction in organic light-emitting devices. IEEE Trans Nanotechnol 18, 1057-1062 (2019).

21. Bi YG, Feng J, Liu YS, Li YF, Chen Y et al. Surface plasmonpolariton mediated red emission from organic light-emitting devices based on metallic electrodes integrated with dual-periodic corrugation. Sci Rep 4, 7108 (2014).

22. Xu M, Feng J, Liu YS, Jin Y, Wang HY et al. Effective and tunable light trapping in bulk heterojunction organic solar cells by employing Au-Ag alloy nanoparticles. Appl Phys Lett 105, 153303 (2014).

23. Huang CY, Zhang XP, Wang JS, Hong CY. Toward electrically pumped polymer lasing: light-emitting diodes based on microcavity arrays of distributed bragg gratings. Adv Opt Mater $\mathbf{6}$, 1800806 (2018).

24. Liang HW, Hsu HC, Wu JN, He XF, Wei MK et al. Corrugated organic light-emitting diodes to effectively extract internal modes. Opt Express 27, A372-A384 (2019).

25. Garcia RF, Zeng L, Khadir S, Chakaroun M, Fischer APA et al. Enhanced electroluminescence of an organic light-emitting diode by localized surface plasmon using Al periodic structure. $J$ Opt Soc Am B 33, 246-252 (2016).

26. Lindquist NC, Luhman WA, Oh SH, Holmes RJ. Plasmonic nanocavity arrays for enhanced efficiency in organic photovoltaic cells. Appl Phys Lett 93, 123308 (2008).

27. Deng LL, Yang JQ, Zhan N, Yu TY, Yu HT et al. High-performance solution-processed white organic light-emitting diodes based on silica-coated silver nanocubes. Opt Lett 44, 983-986 
(2019).

28. Andrew $P$, Barnes WL. Energy transfer across a metal film mediated by surface plasmon polaritons. Science 306, 1002-1005 (2004).

29. Liu YS, Guo S, Yi FS, Feng J, Sun HB. Highly flexible organicinorganic hybrid perovskite light-emitting devices based on an ultrathin Au electrode. Opt Lett 43, 5524-5527 (2018).

30. Bi YG, Feng J, Li YF, Jin Y, Liu YF et al. Enhanced efficiency of organic light-emitting devices with metallic electrodes by integrating periodically corrugated structure. Appl Phys Lett 100,
053304 (2012).

\section{Acknowledgements}

This work was supported by the National Key Research and Development Program of China and the National Natural Science Foundation of China (NSFC) under Grants No. 2020YFA0715000 and No. 61825402.

\section{Competing interests}

The authors declare no competing financial interests. 